\title{
Uncertain Environmental Footprint of Current and Future Battery Electric Vehicles
}

\author{
Brian Cox, ${ }^{*}{ }^{\dagger} \odot$ Christopher L Mutel, ${ }^{\dagger}$ C Christian Bauer, ${ }^{\dagger}$ Angelica Mendoza Beltran, ${ }^{\dagger}$ \\ and Detlef P. van Vuuren ${ }^{\S}$ \\ ${ }^{\dagger}$ Paul Scherrer Institut Laboratory for Energy Systems Analysis PSI, Villigen 5232, Switzerland \\ ${ }^{\ddagger}$ Leiden University Institute of Environmental Sciences (CML), Leiden 2300, Netherlands \\ ${ }^{\S}$ PBL Netherlands Environmental Assessment Agency, The Hague 2594, Netherlands
}

\section{Supporting Information}

ABSTRACT: The future environmental impacts of battery electric vehicles (EVs) are very important given their expected dominance in future transport systems. Previous studies have shown these impacts to be highly uncertain, though a detailed treatment of this uncertainty is still lacking. We help to fill this gap by using Monte Carlo and global sensitivity analysis to quantify parametric uncertainty and also consider two additional factors that have not yet been addressed in the field. First, we include changes to driving patterns due to the introduction of autonomous and connected vehicles. Second, we deeply integrate scenario results from the IMAGE integrated assessment model into our life cycle database to include the impacts of changes to the electricity sector on the environmental burdens of producing and recharging future EVs. Future EVs are expected to have $45-78 \%$ lower climate change impacts than current EVs. Electricity used for charging is the largest source of variability in results, though vehicle size, lifetime, driving patterns, and battery size also strongly contribute to variability. We also show that it is imperative to consider changes to the electricity sector when calculating upstream impacts of EVs, as without this, results could be overestimated by up to $75 \%$.

\section{INTRODUCTION}

The environmental impacts of current battery electric vehicles (here referred to as EVs) are a common topic in the current literature. On the basis of this work, it is well-known that the largest contributors to the environmental impacts of EVs typically include the production of the electricity used to charge the EV and the production of the EV, especially the battery. ${ }^{1,2}$ At the same time, the exact results vary widely depending on (a) the input assumptions that determine the amount of energy consumed per kilometer, such as vehicle mass, efficiency, driving patterns, and auxiliary energy demand, ${ }^{2-4}$ (b) the source of the electricity used for recharging, ${ }^{1,5}$ and (c) input assumptions regarding the size and lifetime of the battery and the vehicle itself. ${ }^{1-3}$ Additionally, the uncertain amount of energy consumed during the lithium ion battery production process can also have a strong impact on the results. ${ }^{6-8}$

Although much progress has been made in understanding the current environmental impacts of EVs, it is the performance of future EVs that is most relevant for policy makers, scientists and transport planners, as these are the vehicles that are expected to make up significant portions of the future passenger vehicle fleet. Studies that address this topic point out that assessment of future EVs face the same difficulties regarding input assumptions as the assessment of current EVs, with the added difficulty of uncertain future technology performance and driving patterns. ${ }^{1,4,5}$ One major potential change in the future environmental impacts of passenger vehicles is the introduction of autonomous driving capabilities, which could have a strong influence on vehicle energy consumption ${ }^{9}$ and has not yet been included in life cycle assessment studies.

In this work, we address all of the above uncertainties using a Life Cycle Assessment (LCA) modeling framework. We quantify the impact of uncertain input parameters and key life cycle inventories on the environmental performance of current (production year 2017) and future (production year 2040) EVs. We integrate Monte Carlo analysis with driving cycle simulation to calculate vehicle energy consumption and endogenously include the impacts that connected and autonomous vehicles may have on energy demand, such as traffic smoothing, eco-driving, and platooning.

An additional major uncertainty that we address is the background energy system that will be used to build the future $\mathrm{EV}$ and relevant infrastructure, which can also have strong

Received: January 15, 2018

Revised: March 18, 2018

Accepted: March 23, 2018

Published: March 23, 2018 
Table 1. Selected Input Value Ranges for Current and Future Electric Vehicles ${ }^{a}$

\begin{tabular}{|c|c|c|c|c|c|c|c|c|}
\hline \multirow[b]{2}{*}{ vehicle type } & \multirow[b]{2}{*}{ variable } & \multirow[b]{2}{*}{ units } & \multicolumn{3}{|c|}{ current } & \multicolumn{3}{|c|}{ future } \\
\hline & & & mode & $\min$ & $\max$ & mode & $\min$ & $\max$ \\
\hline \multirow{4}{*}{ glider } & vehicle lifetime & $\mathrm{km}$ & 150000 & 100000 & 300000 & 150000 & 100000 & 400000 \\
\hline & base mass & $\mathrm{kg}$ & 1100 & 700 & 1600 & 1062 & 651 & 1600 \\
\hline & lightweighting potential & $\%$ & 5 & 0 & 20 & 10 & 0 & 25 \\
\hline & aerodynamic drag coefficient & & 0.31 & 0.3 & 0.34 & 0.295 & 0.264 & 0.35 \\
\hline \multirow{6}{*}{$\begin{array}{l}\text { powertrain } \\
\text { battery }\end{array}$} & rolling resistance coefficient & & 0.010 & 0.007 & 0.012 & 0.009 & 0.006 & 0.012 \\
\hline & auxiliary power demand & $\mathrm{W}$ & 636 & 458 & 783 & 604 & 275 & 783 \\
\hline & efficiency & $\%$ & 80 & 75 & 85 & 81 & 75 & 88 \\
\hline & mass & $\mathrm{kg}$ & 350 & 150 & 600 & 350 & 150 & 600 \\
\hline & charge efficiency & & 0.9 & 0.85 & 0.94 & 0.915 & 0.85 & 0.96 \\
\hline & discharge efficiency & & 0.9 & 0.85 & 0.94 & 0.915 & 0.85 & 0.96 \\
\hline \multirow{9}{*}{ autonomous driving } & cell energy density & $\mathrm{kWh} / \mathrm{kg}$ & 0.2 & 0.15 & 0.25 & 0.4 & 0.25 & 0.5 \\
\hline & cell share of total battery mass & & 0.6 & 0.55 & 0.65 & 0.65 & 0.6 & 0.7 \\
\hline & cell production electricity & $\mathrm{kWh} / \mathrm{kg}$ cell & 24 & 15 & 30 & 15 & 8 & 24 \\
\hline & lifetime & $\mathrm{km}$ & 150000 & 80000 & 300000 & 150000 & 120000 & 350000 \\
\hline & calculation power & $\mathrm{W}$ & & & & 100 & 0 & 200 \\
\hline & aerodynamic penalty & $\%$ & & & & 0 & 0 & 2 \\
\hline & electronics mass & $\mathrm{kg}$ & & & & 5 & 0 & 10 \\
\hline & driving cycle smoothing & alpha & & & & 0.90 & 0 & 0.98 \\
\hline & platooning drag reduction & & & & & $10 \%$ & 0 & $40 \%$ \\
\hline
\end{tabular}

influence on the environmental burdens caused by EVs. ${ }^{10-12}$ This is much more involved than simply assuming a different electricity source is used to charge future EVs as is common in the literature; ${ }^{1,2}$ we make systematic changes to the LCA database so that the whole EV production chain is consistent with the electricity scenario used. ${ }^{12}$

\section{METHODS}

In the main body of the paper, we focus on three novel aspects of our work:

1. The quantification of current and future electric vehicle performance including parameter uncertainty.

2. The estimation of energy consumption of future autonomous vehicles.

3. The creation of future LCA databases for two different electricity scenarios.

A complete description of the methods as well as the calculation files can be found in the Supporting Information.

2.1. Performance of Electric Vehicles. Our EV energy consumption model is based on the work of Bauer et al. ${ }^{5}$ who simulate the operation of a vehicle following a fixed velocity versus time curve. The mechanical energy demand required by the vehicle to follow the velocity profile can be estimated based on the vehicle mass, frontal area, aerodynamic drag, and rolling resistance. The Tank to Wheel ( $\mathrm{TtW})$ energy consumption of the vehicle can then be calculated using input values for powertrain and battery efficiencies, as well as auxiliary power demands, such as lighting, navigation, and heating and air conditioning. Heating and air conditioning energy consumption are estimated based on simple approximations for monthly average temperature control demands for three typical climate types and correspond well with real world operating data of EVs. ${ }^{13}$ See the Supporting Information for more information.

The model considers vehicle sizes ranging from mini to luxury car, with vehicle mass as an uncertain parameter. The most likely vehicle size in our model is comparable to popular EVs such as the Renault Zoe and the Nissan Leaf with curb masses in the range of $1400-1500 \mathrm{~kg}$ and battery packs with $30-50 \mathrm{kWh}$ capacity. We also perform the calculation for small, medium, and large EVs separately in the Supporting Information.

We calibrate our input assumptions for current EVs using literature values (see Figure SI4), and include the potential future change for each input parameter separately based on literature review and own calculations. When estimating future performance of EVs, we include a worst case value that is similar to current performance, and a best case value that is considers optimistic continuation of historic rates of improvement. The most important of these assumptions, as well as other assumptions for LCA are presented in Table 1, whereas all input assumptions, their sources, and description are available in the Supporting Information.

Monte Carlo analysis is used to calculate uncertain life cycle inventories for current and future EVs. With this approach, we calculate the potential performance of tens of thousands of current and future EV configurations, each with different values for all parameters selected randomly according to their defined probability distribution. We account for the fact that many variables are correlated by carefully specifying which variables are sampled as input parameters and which variables are calculated as a result of these input parameters. For example, there is strong correlation between vehicle mass, energy consumption, range and battery mass. We solve this problem by only defining vehicle design characteristics such as glider mass, battery mass, aerodynamic drag coefficient, and component efficiencies and calculating vehicle energy consumption and range on the basis of these values. This ensures that every EV configuration that could be possibly determined by the model is feasible.

2.2. Energy Consumption of Autonomous and Connected Vehicles. Autonomous vehicles have the potential to reduce road congestion, automate eco-driving, improve traffic flow at intersections, and optimize speed profiles based on knowledge of the road ahead. ${ }^{9,14-19}$ We group all of these driving patterns under the heading "traffic smoothing". Though 

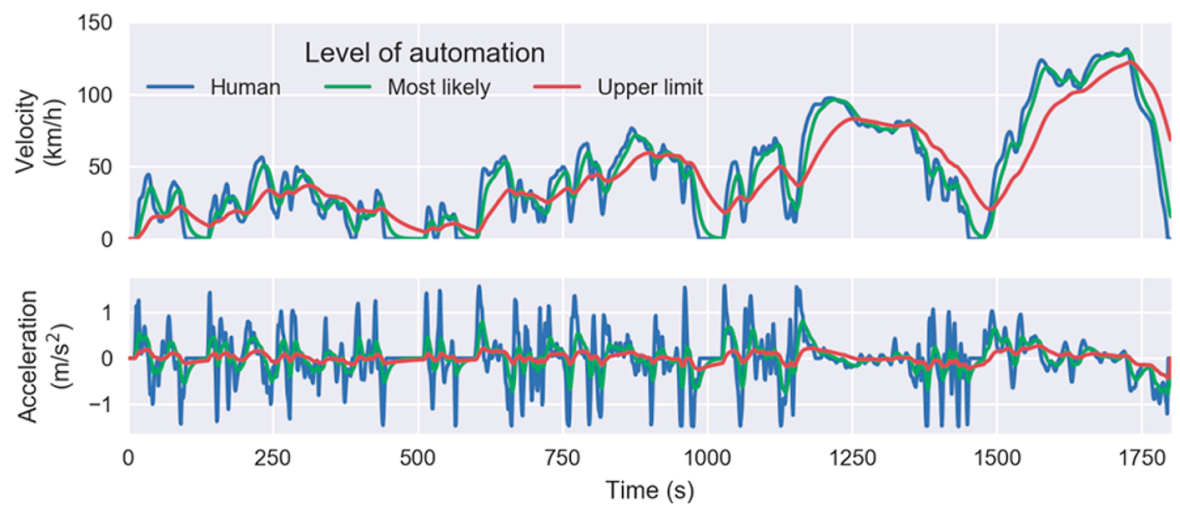

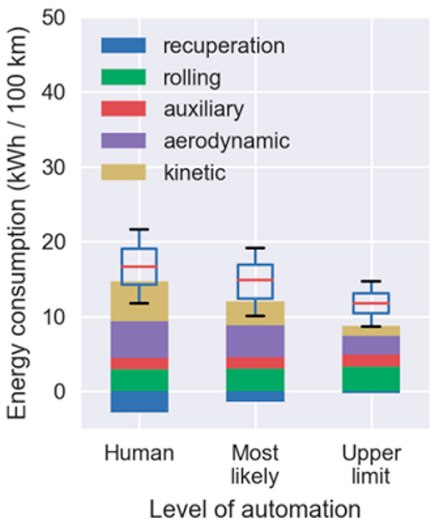

Figure 1. Comparison of human and autonomously driven future electric vehicles. The left panels show driving cycle smoothing results for the Worldwide harmonized Light vehicles Test Cycle with three levels of traffic smoothing; the top panel shows velocity, while the bottom panel shows acceleration versus time. The right panel shows future EV tank to wheel energy consumption. The boxes contain $50 \%$ of the results, whereas the whiskers show the 5th and 95th percentiles and the red line shows the median result.

the aforementioned studies provide estimates for potential autonomous vehicle energy savings, their results cannot be used directly because energy savings depends on the specific properties of the vehicle used for the analysis. The solution is to include the impacts of traffic smoothing endogenously in our energy consumption model. We estimate the impacts on driving patterns by applying exponential smoothing to existing driving cycles. Exponential smoothing of time series data can be calculated using the equation: $v_{t}=(1-\alpha) V_{t}+\alpha v_{t-1}$, where $v$ is the actual velocity at time $t, V$ is the target velocity, and $\alpha$ is the damping parameter ranging between zero and one. As the smoothing parameter is highly uncertain, we assume a uniform distribution between 0 and 0.98 , with a most likely value of 0.9 . See Figure 1 for driving cycle smoothing results.

Autonomous and connected vehicles are also expected to be able to form platoons in order to reduce aerodynamic drag. We take use a mode drag coefficient reduction value of $10 \%$, with a range of 0 to $40 \%$ reduction based on the findings of Zabat et $\mathrm{al}^{20}$ as an input for the Monte Carlo analysis. We further include higher auxiliary power demand for autonomous vehicles to represent the energy consumed by the computer used to control the vehicle and the communications devices required. We estimate this continuous power demand to be up to $200 \mathrm{~W}$, which is similar to the energy consumption of a powerful desktop computer. We also estimate the weight of the additional electrical and sensing devices required for autonomous driving to be up to $10 \mathrm{~kg}$, and introduce a small aerodynamic penalty of up to $2 \%$ increase in the aerodynamic drag coefficient due to sensor positioning.

2.3. Life Cycle Assessment. The functional unit of the study is one vehicle kilometer (vkm) averaged over the entire life cycle of the vehicle (most likely value is $150000 \mathrm{~km}$ ). Life cycle inventories (LCI) for electric vehicle components are generally taken from ecoinvent v3.4, with battery data sets based on the work of Ellingsen et al. ${ }^{6}$ Vehicles are assumed to have global average production chains and the electricity used for charging is considered an uncertain input parameter. We include all electricity markets in the ecoinvent 3.4 database, with their probability of selection being that markets share of global electricity production. The most likely value is set to be the global average electricity mix. Detailed LCA methods, LCI data, as well as results for other electricity sources are available in the Supporting Information.
We modify the ecoinvent database for the LCA calculation of $2040 \mathrm{EVs}$ to consider the future development of the electricity sector using scenario data from the IMAGE Integrated Assessment Model. $^{21}$ IMAGE provides a detailed description of the possible development of the future energy system within a wider context of drivers of environmental change. For this study, we use the IMAGE implementation of the Shared Socioeconomic Pathways, a set of community scenarios used by many modeling teams. ${ }^{22}$ More specifically, we use the "Middle of the Road" scenario, SSP2 (Baseline), and a variant of this scenario (ClimPol) in which an aggressive climate policy is introduced that limits the greenhouse gas concentration to 450 ppm of $\mathrm{CO}_{2}$ eq in 2100 , consistent with a likely probability of achieving the $2{ }^{\circ} \mathrm{C}$ target. In both cases, we use the results for 2040. The global average electricity mix for each scenario can be examined in Figure SI11.

Using the Wurst python package, we modify the relevant electricity sector entries in the ecoinvent database using information from the IMAGE scenario results for 26 global regions. Two main changes are made: (1) Electricity mixes are changed in each ecoinvent location using IMAGE results. (2) All fossil, biomass and nuclear power plant data sets are modified by directly taking over IMAGE results for criteria pollutant emissions and scaling all other exchanges according to future efficiency improvements defined by the IMAGE results. All other production technologies are left unchanged, though the electricity used throughout their supply chains is also modified, based on the changes described above. LCI data sets for electricity generation technologies with carbon capture and storage are not available in ecoinvent, so we added data sets from previous work of the authors. ${ }^{23}$ More information on our work developing of future versions of LCA databases can be found in Mendoza Beltran, Cox, Mutel, Vuuren, Vivanco, Deetman, Edelenbosch, Guinée, and Tukker. ${ }^{12}$

We calculate LCA results for current and future EVs with the original ecoinvent 3.4 database (Current) as well as the future EVs with each of the two modified databases, which can be considered to represent most likely (Baseline), and best case scenarios (ClimPol).

\section{RESULTS}

Driving patterns and energy consumption results for future human driven and autonomous vehicles are compared in Figure 1. Two levels of automation, most likely and upper limit, are 
shown, calculated with input values for traffic smoothing, platooning, and additional auxiliary energy consumption from Table 1 . The panels on the left show velocity and acceleration time series data. The "Human" scenario is the Worldwide harmonized Light vehicles Test Cycle (WLTC), whereas the autonomous vehicle scenarios are exponential smoothing results with the WLTC as an input. The bar plot on the right shows tank-to-wheel energy consumption for the three scenarios. In the most likely scenario acceleration peaks are already significantly reduced, though the velocity profile remains very similar to that of a human driver. In the upper limit scenario, the velocity profile is very much smoothed and acceleration peaks are minimized.

Kinetic energy demand is greatly reduced because of the lower acceleration requirements, but this also reduces the energy recoverable by recuperative braking, and some of the benefits of vehicle lightweighting. Traffic smoothing is expected to reduce vehicle energy consumption by roughly $13 \%$ in the most likely case, with savings up to $20 \%$ in the upper limit compared to human drivers. The energy consumption savings due to platooning are found to be $12 \%$ in the most likely case and up to $49 \%$ in the upper limit. Energy consumption due to auxiliaries is not expected to increase significantly, though it could increase by up to $5 \%$ in the upper limit. In total, autonomous and connected vehicles are expected to have $11 \%$ lower energy consumption than human drivers, with a maximum reduction of $29 \%$.

These results are in line with other published values for the expected energy savings of autonomous and connected vehicles. ${ }^{14}$ This appears to be a reasonable method for integrating the uncertain impacts of autonomous and connected vehicles on future driving patterns with vehicle energy consumption simulation. The most likely result shows how an energy-optimized autonomous vehicle could operate in traffic situations that include human drivers without greatly impacting the traffic flow, whereas the upper limit could represent a world where nearly all vehicles on the road are autonomous and cooperate to minimize energy consumption, as kinetic and aerodynamic energy demands approach their physical minimum limits in this scenario.

In Figure 2, we show the climate change impacts per vehicle kilometer (vkm) traveled by the EVs. Results for other environmental impact categories can be found in the Supporting Information. The top bar shows results for current EVs, whereas the bottom three bars show results for future EVs, calculated with the three versions of the background database. The stacked bar chart shows the most likely result (mode), calculated using the most likely value for every input parameter (see the Supporting Information section 1.1 for complete input values), and global average electricity used for battery charging. The bar is broken down into four categories, representing different stages of the EV life cycle. Road refers to the production and maintenance of the road, Battery refers to the production and end-of-life treatment of the battery, including replacement batteries for EV require them (see the Supporting Information). Rest of car refers to the production, maintenance, and end-of-life of all components in the car except for the battery. Energy chain refers to the entire chain of electricity generation, transmission, voltage conversion and charging infrastructure to charge the battery. All electricity grid mixes in the world are included as an uncertain input parameter and selected based on their contribution share to total global electricity production according to the production volumes in

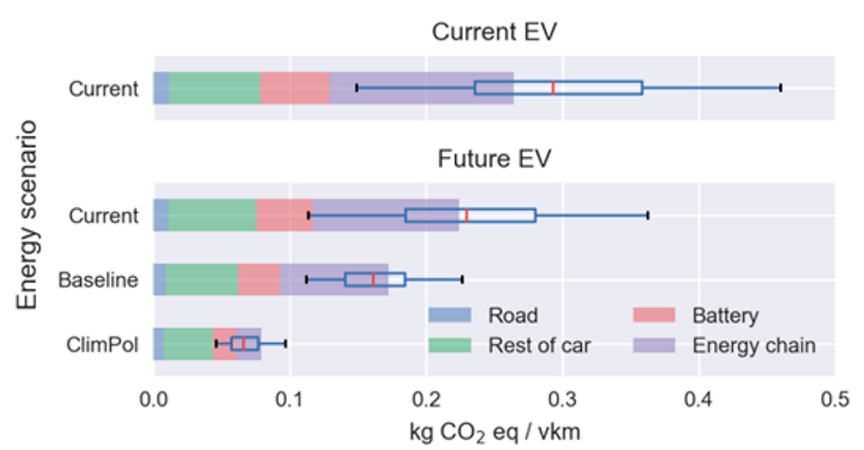

Figure 2. Life cycle climate change contribution of current and future electric vehicles per kilometer driven. The boxes contain $50 \%$ of the results, whereas the whiskers show the 5th and 95th percentiles and the red line shows the median result. $Y$ axis labels show the background database that was used for the calculation: ecoinvent uses the current electricity scenario, whereas Baseline and ClimPol show middle-of-the-road and aggressive climate policy scenarios for the future electricity sector, respectively. The emission categories are explained in the text.

ecoinvent 3.4. The boxplots show the uncertainty of the results. The boxes contain $50 \%$ of the results for each scenario, while the whiskers show the 5th and 95th percentiles and the red line shows the median result. We note that the median and the most likely value (mode) are not necessarily the same because of the asymmetrical nature of the input parameter uncertainty distributions (See Figure SI23).

We find that current EVs operating with global average electricity have mean life cycle greenhouse gas emissions of 316 $\mathrm{g} \mathrm{CO}_{2} \mathrm{eq} / \mathrm{km}$ (mode $277 \mathrm{~g} \mathrm{CO}_{2} \mathrm{eq} / \mathrm{km}$, standard deviation 103 $\mathrm{g} \mathrm{CO}_{2} \mathrm{eq} / \mathrm{km}$ ). The large standard deviation is primarily due to the large variation in the carbon intensity of the electricity used to charge the batteries. Without uncertainty regarding the electricity source selection, the standard deviation is reduced to below $50 \mathrm{~g}$ of $\mathrm{CO}_{2} \mathrm{eq} / \mathrm{km}$. Our mean values for current EVs are comparatively high compared to recent findings in the literature, which is explained by our use of global average electricity, which has a climate change impact of $839 \mathrm{~g}$ of $\mathrm{CO}_{2}$ eq/kWh, and our attempt to include more realistic EV energy consumption values. We show calibration results for our energy consumption model in section 1.1 .7 of the Supporting Information. If low carbon sources of electricity are used, such as wind, hydro, or solar photovoltaic, the impact reduces to $130-50 \mathrm{~g}$ of $\mathrm{CO}_{2} \mathrm{eq} / \mathrm{km}$ (standard deviation $31 \mathrm{~g}$ of $\mathrm{CO}_{2}$ eq $/ \mathrm{km}$ ). See section 2.7 of the Supporting Information for results with different electricity production technologies, and section 2.3 of the Supporting Information for results for different vehicle size categories.

When comparing current and future EVs built and operated with the current electricity sector, we see that roughly $20 \%$ improvement is expected due to improvements of the EVs themselves. The most significant improvement, however, is found when changing the electricity sector used to produce and operate the future EVs. With the Baseline scenario, the climate change impact of the future EVs has a mean of $172 \mathrm{~g}$ of $\mathrm{CO}_{2}$ $\mathrm{eq} / \mathrm{km}$ (mode $180 \mathrm{~g}$ of $\mathrm{CO}_{2} \mathrm{eq} / \mathrm{km}$, standard deviation $38 \mathrm{~g}$ of $\mathrm{CO}_{2} \mathrm{eq} / \mathrm{km}$ ); the ClimPol scenario leads to a mean result of 70 $\mathrm{g}$ of $\mathrm{CO}_{2} \mathrm{eq} / \mathrm{km}$ (mode $80 \mathrm{~g}$ of $\mathrm{CO}_{2} \mathrm{eq} / \mathrm{km}$, standard deviation $17 \mathrm{~g}$ of $\left.\mathrm{CO}_{2} \mathrm{eq} / \mathrm{km}\right)$. Compared to future EVs produced and operated with the current electricity system, the Baseline and ClimPol scenarios show life cycle greenhouse gas (GHG) emission reductions of $15 \%$ and $65 \%$, respectively. Compared 

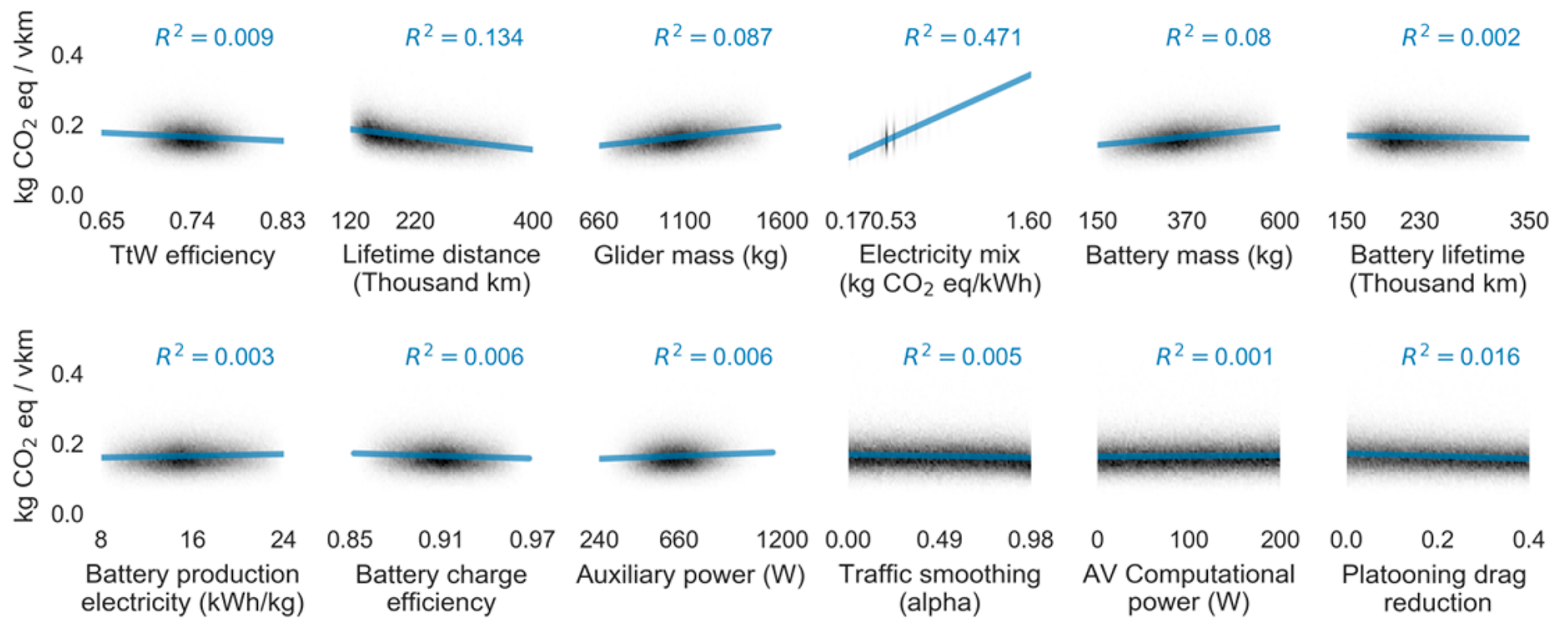

Battery mass $(\mathrm{kg})$

(Thousand $\mathrm{km}$ )
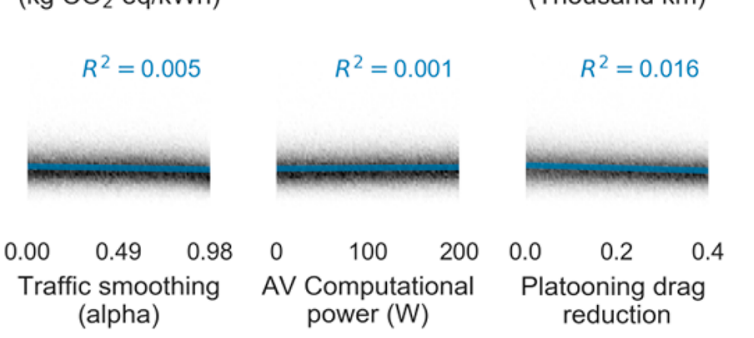

reduction

Figure 3. Monte Carlo results for climate change versus selected input parameters. Results are for future electric vehicles with 2040 Baseline electricity scenario. $\mathrm{AV}=$ autonomous vehicle, $\mathrm{TtW}=$ tank to wheel. Blue line indicates linear regression result.

to current EVs, the Baseline and ClimPol scenarios show reductions of 30 and $72 \%$, respectively.

While the majority of these improvements are due to the decreasing impacts of electricity production for battery charging, the changes in the upstream impacts from electricity production on the rest of the life cycle are also significant. We include results for EV production in the Suporting Information: Figures SI5-SI12 show the environmental impact associated with current and future EV production. Compared to current EVs, future EVs are expected to have lower production impacts for nearly all indicators due to a combination of the reduced energy consumption of battery production and the improvements to the electricity sector. The life cycle GHG emissions caused by future EV production in the ClimPol scenario are less than half of those for current EVs. This corresponds to a reduction of EV production and end-of-life burdens from $106 \mathrm{~g}$ of $\mathrm{CO}_{2} \mathrm{eq} / \mathrm{km}$ for future EVs built with the current electricity mix to $83 \mathrm{~g}$ of $\mathrm{CO}_{2} \mathrm{eq} / \mathrm{km}$ for the Baseline scenario and $54 \mathrm{~g}$ of $\mathrm{CO}_{2} \mathrm{eq} / \mathrm{km}$ for the ClimPol scenario. This means that neglecting the development of the electricity sector used to build the vehicle and infrastructure are overestimating the GHG emissions by $13 \%$ in the Baseline scenario and $75 \%$ in the ClimPol scenario.

Results for mineral depletion and human toxicity are driven by the material inputs of batteries; these results do not change significantly for future EVs because battery mass per vehicle is expected to remain relatively constant in the future. If future EVs do turn out to have smaller batteries than today's vehicles, impacts in these categories will likely decrease.

The results presented in the main body of the paper do not include the uncertainty in the background database, though we include it in the Supporting Information section 2.8. When background uncertainty is also included, mean results increase by $21-26 \mathrm{~g}$ of $\mathrm{CO}_{2} \mathrm{eq} / \mathrm{km}$ due the difference between the mean and mode values in the underlying uncertainty distributions. Static calculations in the database are performed with the mode, whereas uncertainty calculations are centered on the mean value for each uncertain parameter. Including the uncertainty of the background database increases the standard deviation of the overall result by less than $10 \mathrm{~g}$ of $\mathrm{CO}_{2} \mathrm{eq} / \mathrm{km}$.

We next examine the main drivers for the foreground variability in the results. Figure 3 is a hexbin plot representing the life cycle GHG emissions per vehicle kilometer versus the variation in selected input parameters for future EVs calculated with the Baseline scenario. Results for current EVs and future EVs for other background scenarios are included in the Supporting Information. The shade of the plot indicates the number of Monte Carlo iterations that produced a result with that value, with darker regions indicating more common outcomes. Larger slopes and $R^{2}$ values for a parameter indicate that the results are more sensitive to that parameter. We also perform global sensitivity analysis on the model results. Figure 4 shows results for the ten input parameters that contribute

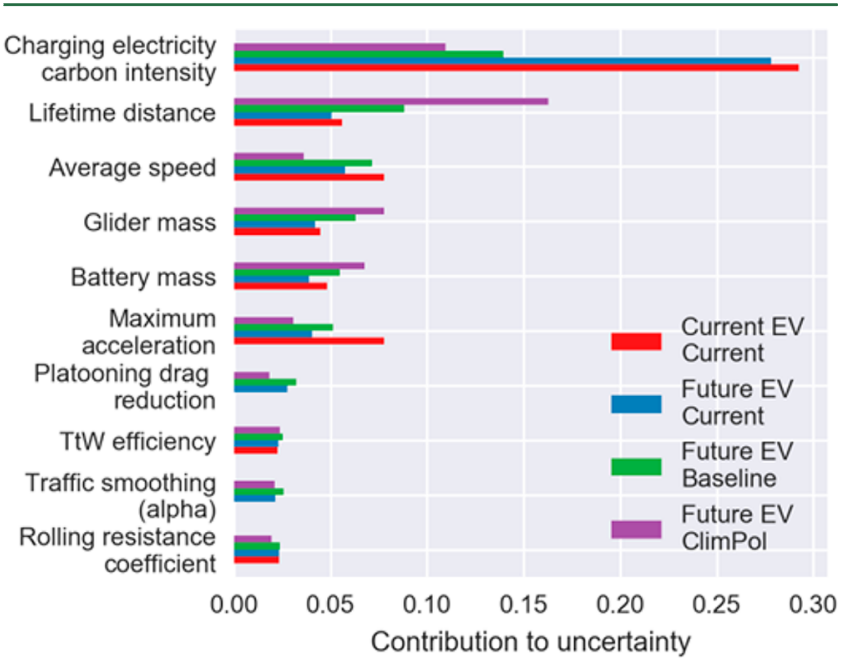

Figure 4. Global sensitivity analysis results for climate change results, 10 largest contributors.

most to climate change result variability; results for all input variables and environmental impact categories can be found in the Supporting Information.

Variance in climate change impacts are most strongly driven by the variability of the carbon intensity of the electricity used to charge the EVs. This trend is less pronounced in future scenarios with cleaner electricity overall, as there is both lower variability in the carbon intensity of the electricity production, but also the electricity production contributes less overall to the total life cycle impacts. See Supporting Information Section 2.7 for results with different charging electricity sources. 
Especially for these scenarios with lower overall impacts because of electricity production, the next most important parameter is found to be the lifetime distance traveled by the vehicle. This is because it directly influences the share of the EV production burdens that are allocated to each kilometer traveled by the vehicle. We find that vehicles that travel further over their lifetimes are expected to have lower impacts per kilometer traveled. Thus, innovations such as car sharing that are expected to increase the utilization of existing vehicle infrastructure are likely to have positive impacts in this regard.

The next group of input parameters with strong impact on the results determine the mass of the vehicle and the size of the battery. These parameters not only influence the production impacts of the vehicle but also the energy consumption of the vehicle, as this depends strongly on vehicle weight. We include separate vehicle size categories in the results in the Supporting Information. Compared to medium-sized vehicles, small and large vehicles are found to have roughly $20 \%$ lower and higher impacts per kilometer, respectively. EVs with the largest batteries are found to have up to $40 \%$ higher life cycle GHG emissions per kilometer compared to similar vehicles with smaller batteries.

The driving pattern of the vehicle, here quantified by average speed, $\mathrm{m}$ acceleration, platoon drag reduction, and traffic smoothing parameters, is also found to have large impact on the result, with more aggressive acceleration and higher average speeds yielding higher climate change contributions. The introduction of autonomous and connected vehicles is found to decrease sensitivity of results to driving cycle choice.

\section{DISCUSSION}

In this study, we examine the environmental impacts of current and future electric vehicles with a focus on parametric uncertainty and the developments of the future electricity system. We find that the electricity used for charging is the largest source of variability in results, though vehicle size, lifetime, driving patterns, and battery size also contribute to variability in results. We also develop a method of including the energy savings potential of future autonomous and connected vehicles in our model and find that this new technology is most likely to result in roughly $10 \%$ energy savings, though energy savings could be up to $30 \%$ with ideal technology performance. Future electric vehicles are likely to have roughly $20 \%$ lower environmental impacts compared to current vehicles based on technological improvements alone. However, when also considering the future improvement of the electricity sector, these improvements are in the range of $30-70 \%$ for baseline and climate policy scenarios. This is an important conclusion that must be considered when making policy in the mobility sector as use of current results as a proxy for future technologies would be highly misleading.

It is common practice when performing LCA of future technologies such as electric vehicles to assume that the background database used for infrastructure production can be considered constant and that the future electricity mix needs only be considered for direct consumption. We find that neglecting the improvement of the future electricity system when calculating the upstream impacts of $\mathrm{EV}$ production changes the results by $23-52 \mathrm{~g}$ of $\mathrm{CO}_{2} \mathrm{eq} / \mathrm{km}$. This is an error of over two standard deviations for the ClimPol scenario! Thus, especially in future scenarios where renewable electricity is used to charge the EVs, it is extremely important to consider also the development of the electricity sector, as the upstream impacts due to electricity production can significantly impact results.

Despite the advances we made in understanding and including uncertainties in the future impacts of EVs, there are some limitations in our study that require further consideration. First, we have used current life cycle inventories for the modeling of future technologies and changed only key parameters. This could especially be important for battery production and electric vehicle end-of-life treatment as significant future development is expected in both of these areas that could change results significantly. Second, we have focused our uncertainty quantification on input parameters and have not modified the uncertainty values defined in the ecoinvent processes used. Though we show in the Supporting Information section 2.8 that foreground parametric uncertainty dominates background database uncertainty for the climate change impact category, more effort should be expended to improve uncertainty values in the ecoinvent database to confirm that this is the case, especially for other environmental impact categories.

Third, the approach to include the energy-related impacts of autonomous and connected vehicles in our model is relatively simple. Though the actual energy savings are uncertain, we are confident that we have managed to define the likely upper and lower bounds of these parameters and the energy savings found are consistent with other studies. ${ }^{9,14}$ Furthermore, we have not considered any other implications of the rise of autonomous and connected vehicles, such as car sharing or increased distances traveled per person. Fourth, our modifications to the ecoinvent database consider only future electricity production and are limited to only two scenarios. Future work should attempt to include other important aspects such as changes in raw materials production, recycling, heat production and consumption, and transportation of goods and should also develop methods to quantify the uncertainty of these scenarios. Finally, we have not considered uncertainty related to choices of system model or uncertainty in impact assessment methods used, though these are likely substantial. We refer the reader to the Supporting Information for a more complete discussion of the limitations to this study.

\section{ASSOCIATED CONTENT}

\section{S Supporting Information}

The Supporting Information is available free of charge on the ACS Publications website at DOI: 10.1021/acs.est.8b00261.

Extended description of the methods used and results. (PDF)

Input assumptions for the calculation files (XLSX)

Calculations files that can be used to repeat our work: Additional data sets.xlsx is an excel file that contains additional LCI data that were used in the calculations; Calculation file.ipynb is the main jupyter notebook that was used to perform the calculations (also available as.html file for readers that do not use jupyter notebooks); Initialize_notebook.ipynb is a helper file used by calculation file.ipynb to initialize variables and define functions to improve readability (also available as html file for readers that do not use jupyter notebooks); Global Sensitivity Analysis.ipynb is a helper file for Calculation file.ipynb that performs the global sensitivity analysis on results (also available as .html file for readers that do not use jupyter notebooks) (ZIP) 


\section{AUTHOR INFORMATION}

\section{Corresponding Author}

*E-mail: brian.cox@psi.ch.

\section{ORCID $\odot$}

Brian Cox: 0000-0002-4531-6709

Christopher L Mutel: 0000-0002-7898-9862

\section{Author Contributions}

B.C. performed all calculations and prepared the manuscript. C.M. greatly contributed to the calculation framework and edited the manuscript. C.B. provided guidance regarding the modeling of EVs and edited the manuscript. A.M.B. greatly contributed to the generation of the future versions of the LCA database and edited the manuscript. D.P.v.V. provided detailed knowledge of the IMAGE model and edited the manuscript.

\section{Notes}

The authors declare no competing financial interest.

\section{ACKNOWLEDGMENTS}

The authors thank Prof. Alexander Wokaun and Stefan Hirschberg for their comments and guidance, Karin Treyer for her insight into the ecoinvent electricity models, as well as Simon Schneider and Tom Terlouw for their help in data collection. This research was supported by the Swiss Competence Center for Energy Research (SCCER) Efficient Technologies and Systems for Mobility, funded by the Swiss Commission for Technology and Innovation (CTI).

\section{REFERENCES}

(1) Helmers, E.; Weiss, M. Advances and critical aspects in the lifecycle assessment of battery electric cars. Energy and Emission Control Technologies 2017, 5, 1-18.

(2) Nordelöf, A.; Messagie, M.; Tillman, A.-M.; Ljunggren Söderman, M.; Van Mierlo, J. Environmental impacts of hybrid, plug-in hybrid, and battery electric vehicles-what can we learn from life cycle assessment? Int. J. Life Cycle Assess. 2014, 19 (11), 1866-1890.

(3) Ellingsen, L. A.-W.; Singh, B.; Strømman, A. H. The size and range effect: lifecycle greenhouse gas emissions of electric vehicles. Environ. Res. Lett. 2016, 11 (5), 054010.

(4) Hirschberg, S.; Bauer, C.; Cox, B.; Heck, T.; Hofer, J.; Schenler, W.; Simons, A.; Duce, A. D.; Althaus, H.-J.; Georges, G.; Krause, T.; Vaya, M. G.; Ciari, F.; Waraich, R.; Jäggi, B.; Stahel, A.; Frömelt, A.; Saner, D. Opportunities and Challenges for Electric Mobility: An Interdisciplinary Assessment of Passenger Vehicles; PSI, EMPA, ETH: Villigen, Dübendorf, and Zurich, Switzerland, 2016.

(5) Bauer, C.; Hofer, J.; Althaus, H.-J.; Del Duce, A.; Simons, A. The environmental performance of current and future passenger vehicles: Life Cycle Assessment based on a novel scenario analysis framework. Appl. Energy 2015, 157 (1), 871-883.

(6) Ellingsen, L. A.-W.; Majeau-Bettez, G.; Singh, B.; Srivastava, A. K.; Valøen, L. O.; Strømman, A. H. Life Cycle Assessment of a Lithium-Ion Battery Vehicle Pack. J. Ind. Ecol. 2014, 18 (1), 113-124. (7) Dunn, J. B.; Gaines, L.; Kelly, J. C.; James, C.; Gallagher, K. G. The significance of Li-ion batteries in electric vehicle life-cycle energy and emissions and recycling's role in its reduction. Energy Environ. Sci. 2015, 8 (1), 158-168.

(8) Ellingsen, L. A.-W.; Hung, C. R.; Strømman, A. H. Identifying key assumptions and differences in life cycle assessment studies of lithiumion traction batteries with focus on greenhouse gas emissions. Transportation Research Part D: Transport and Environment 2017, 55, 82-90.

(9) Wadud, Z.; MacKenzie, D.; Leiby, P. Help or hindrance? The travel, energy and carbon impacts of highly automated vehicles. Transportation Research Part A: Policy and Practice 2016, 86, 1-18.

(10) Hertwich, E. G.; Gibon, T.; Bouman, E. A.; Arvesen, A.; Suh, S.; Heath, G. A.; Bergesen, J. D.; Ramirez, A.; Vega, M. I.; Shi, L.
Integrated life-cycle assessment of electricity-supply scenarios confirms global environmental benefit of low-carbon technologies. Proc. Natl. Acad. Sci. U. S. A. 2015, 112 (20), 6277-6282.

(11) Ricci, A.; Cosmi, C.; Cuomo, V.; Faberi, S.; Friedrich, R.; Frischknecht, R.; Gaggi, S.; Hirschberg, S.; Karbuz, S.; Krewitt, W.; Loulou, R.; Navrud, S.; Preiss, P.; Scasny, M.; Regermorten, D. V. Final Integrated Report: Policy Use of the NEEDS Results; European Commission: Brussels, Belgium, 2008.

(12) Mendoza Beltran, A.; Cox, B.; Mutel, C.; Vuuren, D. v.; Vivanco, D. F.; Deetman, S.; Edelenbosch, O.; Guinée, J.; Tukker, A., When the Background Matters: Using Scenarios from Integrated Assessment Models (IAMs) in Prospective LCA. J. Ind. Ecol. 2018, submitted.

(13) De Cauwer, C.; Van Mierlo, J.; Coosemans, T. Energy Consumption Prediction for Electric Vehicles Based on Real-World Data. Energies 2015, 8 (8), 8573.

(14) Greenblatt, J. B.; Saxena, S. Autonomous taxis could greatly reduce greenhouse-gas emissions of US light-duty vehicles. Nat. Clim. Change 2015, 5 (9), 860-863.

(15) Fagnant, D. J.; Kockelman, K. Preparing a nation for autonomous vehicles: opportunities, barriers and policy recommendations. Transportation Research Part A: Policy and Practice 2015, 77, $167-181$.

(16) Makarem, L.; Gillet, D. Decentralized Coordination of Autonomous Vehicles at intersections. IFAC Proceedings Volumes 2011, 44 (1), 13046-13051.

(17) Mersky, A. C.; Samaras, C. Fuel economy testing of autonomous vehicles. Transportation Research Part C: Emerging Technologies 2016, 65, 31-48.

(18) Hu, J.; Shao, Y.; Sun, Z.; Bared, J. Integrated vehicle and powertrain optimization for passenger vehicles with vehicle-infrastructure communication. Transportation Research Part C: Emerging Technologies 2017, 79, 85-102.

(19) Stebbins, S.; Hickman, M.; Kim, J.; Vu, H. L. Characterising Green Light Optimal Speed Advisory trajectories for platoon-based optimization. Transportation Research Part C: Emerging Technologies 2017, 82, 43-62.

(20) Zabat, M.; Stabile, N.; Frascaroli, S.; Browand, F., The Aerodynamic Performance of Platoons: A Final Report; California Partners for Advanced Transit and Highways (PATH): Richmond, CA, 1995.

(21) Stehfest, E.; van Vuuren, D.; Bouwman, L.; Kram, T. Integrated Assessment of Global Environmental Change with IMAGE 3.0: Model Description and Policy Applications; Netherlands Environmental Assessment Agency (PBL): The Hague, The Netherlands, 2014.

(22) van Vuuren, D. P.; Riahi, K.; Calvin, K.; Dellink, R.; Emmerling, J.; Fujimori, S.; Kc, S.; Kriegler, E.; O’Neill, B. The Shared Socioeconomic Pathways: Trajectories for human development and global environmental change. Global Environmental Change 2017, 42, 148152.

(23) Volkart, K.; Bauer, C.; Boulet, C. Life cycle assessment of carbon capture and storage in power generation and industry in Europe. Int. J. Greenhouse Gas Control 2013, 16, 91-106. 